\title{
Pseudoexfoliation and sensorineural hearing loss
}

\section{Abstract}

Aims There is increasing evidence that pseudoexfoliation (PXF) not only affects ocular anterior segment structures, but may also be a systemic disease. This study was undertaken to assess the relationship between PXF and sensorineural hearing loss. Methods Patients with PXF were identified from hospital records and underwent complete ocular examination. The sum of pure-tone hearing thresholds measured at 1 , 2 and $3 \mathrm{kHz}\left(\mathrm{HTL}_{1,2,3}\right)$ in each ear was compared with the ISO 7029 standard sexmatched, median age-associated hearing loss summed over the same frequencies

$\left(\mathrm{AAHL}_{1,2,3}\right)$. The proportion of ears with thresholds higher than the ISO 7029 median $\mathrm{AAHL}_{1,2,3}$ on the same side as eyes without PXF was compared with the proportion of ears ipsilateral to eyes with PXF but without glaucoma and similarly the proportion of ears on the same side as eyes with PXF and glaucoma.

Results In total, 69 patients were studied, of whom 39 were male $(56.5 \%)$. The mean age of the male patients was 75.8 years, while that of the female group was 75.1 years. All patients had PXF affecting at least one eye. Overall 101 ears $(73.7 \%)$ had a higher $\mathrm{HTL}_{1,2,3}$ than the ISO 7029 median $\mathrm{AAHL}_{1,2,3}$ which included 56 ears of 78 in the male group (71.8\%) and 45 ears of 59 in the female group (76.3\%). There was no significant difference between the proportion of ears with $\mathrm{HTL}_{1,2,3}$ higher than the ISO 7029 median $\mathrm{AAHL}_{1,2,3}$ on the same side as eyes without PXF, with PXF but not glaucoma and with PXF and glaucoma, in either the male or female groups.

Conclusions A large proportion of patients with PXF have sensorineural hearing loss in comparison to age-matched controls, regardless of whether or not there is associated glaucoma. This finding supports the theory that PXF may be a systemic condition.
M Cahill ${ }^{1}$, A Early ${ }^{1}$, S Stack ${ }^{2}$, AW Blayney ${ }^{2}$ and P Eustace ${ }^{1}$

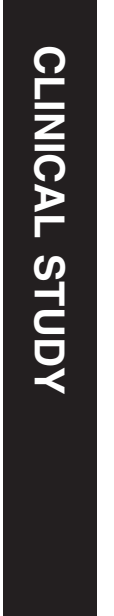

\section{Introduction}

The pseudoexfoliation syndrome (PXF) is characterised by deposits of fibrillar extracellular material on anterior segment structures including the zonules, anterior capsule and trabecular meshwork, and may be associated with raised intraocular pressure in up to $50 \%$ of affected eyes. ${ }^{1-3}$ Recent reports have demonstrated that an identical fibrillopathy to that seen in PXF has been documented pathologically in the basement membranes and extracellular matrices of extraocular orbital tissues, the skin and visceral organs that suggest that PXF is a systemic disease. ${ }^{4-6}$

The organ of Corti is a complex structure in the inner ear, which contains the auditory hair cells, or sound receptors which form the reticular lamina. ${ }^{7}$ These sensory cells are mechanoreceptors characterised by organised sterocilia and sit on a number of support cells which rest on the basilar membrane, a collagen containing element of the organ of Corti. ${ }^{7}$ The tectorial membrane, a soft, fibrillated lamina, the structure of which is characterised by a hydrated matrix stabilised by collagen fibres, covers the sterocilia. ${ }^{8}$ The conversion of the mechanical energy produced by a sound wave to electrical energy resulting in hearing, requires deflection of the stereocilia, induced by a shearing motion between the reticular lamina and the tectorial membrane. This shearing motion is produced by the basilar membrane moving as a result of the travelling sound wave.

Age-associated hearing loss (AAHL), or presbycusis, which is a type of sensorineural hearing loss, can be measured using pure tone audiometry. ${ }^{9,10}$ However, definition of what
${ }^{1}$ Institute of

Ophthalmology University College Dublin Mater Misericordiae Hospital

Dublin, Ireland

${ }^{2}$ Department of Otolaryngology Mater Misericordiae Hospital

Dublin, Ireland

Correspondence:

Mark Cahill

Duke University Eye Center

Erwin Road

PO Box 3802

Durham, NC 27710

USA

Tel: 0019196846611

Fax: 0019196816474

E-mail:cahil005@

mc.duke.edu

Received; 16 January 2001 Accepted: 5 November 2001 
constitutes normal age-associated hearing loss can be difficult. A number of large population-based studies have provided data on the normal reduction in hearing associated with ageing in both otologically normal subjects and also in more typical populations..$^{9-11}$ While both types of population studies used pure tone audiometry summed over a range of frequencies to assess presbycusis, the combination of frequencies used varied. $^{9-11}$ However, in most studies median AAHL has been demonstrated to be less in females than males. ${ }^{9-11}$

The association between hearing loss and glaucoma is not clear with contrasting results from previous reports, depending on the type of glaucoma patients studied and the control populations used. ${ }^{12-15}$ Deafness and glaucoma are associated in a number of congenital syndromes including Alstrom's syndrome and Stickler's syndrome, while two recent reports have demonstrated a familial association between glaucoma and deafness which in one case was associated with abdominal aortic aneurysm. ${ }^{16-18}$ The present study was undertaken to assess the relationship between PXF and sensorineural hearing loss.

\section{Methods}

Consecutive patients with ocular evidence of pseudoexfoliation were identified from the Out-patients Department of the Mater Misericordiae Hospital and invited to participate in the study. Patients were asked about current or previous medical and surgical treatment for glaucoma. Each patient underwent full ophthalmic examination including Snellen visual acuity, slit-lamp biomicroscopy, gonioscopy, applanation tonometry and dilated fundoscopy. Exclusion criteria included history of previous ear surgery, history of tympanic membrane perforation, history of noise exposure including working in a noisy environment and repeated exposure to gunfire, use of ototoxic drugs and concurrent upper respiratory tract infection at the time of the examination.

Pure-tone hearing thresholds were measured at 1, 2 and $3 \mathrm{kHz}$ using air conduction audiometry, and if necessary bone-conduction audiometry, to differentiate between sensorineural and conductive hearing loss. The hearing threshold levels of each ear of all patients were summed over the three frequencies (HTL $\mathrm{H}_{1,2,3}$ ) to the nearest 5 decibels $(\mathrm{dB})$. Testing was started approximately $60 \mathrm{~dB}$ above the expected threshold and continued in descending $10 \mathrm{~dB}$ steps below threshold. Testing was then completed using $5 \mathrm{~dB}$ ascending steps until two of four sounds were heard. Both the air conduction and bone conduction audiometers conformed to recommended standards, as did the quality assurance procedures and the background noise requirements for the testing room. ${ }^{9}$

Control data were provided by the international standard ISO 7029 which gives values of normal ageassociated hearing loss as deviations relative to the median thresholds of young otologically normal subjects. The standard is derived from a meta-analysis of a number of large test populations, but there is some slight variation in the precise meaning of otologically normal. ${ }^{9}$ However, in all test populations, exclusion criteria included an adverse otological history and regular noisy employment. ${ }^{9}$ While the median ageassociated hearing loss has been previously extrapolated up to the age of 80 years, ${ }^{9}$ the original tabulated data, which are limited to values up to and including 70 years of age, were used to calculate the standard deviation of the median age-associated hearing loss. ${ }^{11}$ For comparison, control data on a smaller, more typical population derived from the National Study of Hearing (NSH) were also used..$^{10}$

Each patient's $\mathrm{HTL}_{1,2,3}$ for each ear was plotted on a graph against the ISO 7029 median age-associated hearing loss ( $\pm 1 \mathrm{SD})$, which was similarly summed over the three frequencies of 1,2 and $3 \mathrm{kHz}$ $\left(\mathrm{AAHL}_{1,2,3}\right) .{ }^{9}$ In view of the different pattern of AAHL between males and females, their HTLs were plotted on separate graphs against the ISO 7029 median $\mathrm{AAHL}_{1,2,3}$ and the total proportion of ears above the ISO 7029 median $\mathrm{AAHL}_{1,2,3}$ was determined. In addition, the 'both ears averaged' mean hearing thresholds for these three frequencies $(1,2$ and $3 \mathrm{kHz})$ of 'overall occupational group without noise exposure' subjects of the NSH were plotted on the same graphs. ${ }^{10}$ Median values were not available for the NSH data, but both data sets were normally distributed.

The hearing threshold for a given ear was considered with regard to the ipsilateral eye findings. The proportion of ears with $\mathrm{HTL}_{1,2,3}$ higher than the ISO 7029 median $\mathrm{AAHL}_{1,2,3}$ on the same side as eyes without PXF was compared with the proportion of ears ipsilateral to eyes with PXF, but without glaucoma and similarly the proportion of ears on the same side as eyes with PXF and glaucoma. This three-way comparison was made in both the male and female groups and the significance of any association was determined using the $\chi^{2}$ test.

\section{Results}

In total, 69 patients were studied of whom 39 were male $(56.5 \%)$. The mean age of the male patients was 75.4 years (median 75.6 years; range $59.0-86.3$ years), while that of the female group was 75.1 years (median 77.4 years; range $52.1-84.8$ years). With the exception 
of one patient who had only one ear tested, all patients had bilateral audiometry data resulting in a total of 137 ears studied. All patients had PXF affecting at least one eye and overall 14 eyes had no evidence of PXF (male, seven; 50\%), 29 eyes had PXF but no glaucoma (male, $17 ; 58.6 \%$ ) and 94 eyes had PXF and glaucoma (male, $54 ; 57.4 \%)$.

There were some differences in the age-associated hearing thresholds when the same age groupings in ISO 7029 and the NSH where compared using the 1, 2 and $3 \mathrm{kHz}$ audiometric descriptors. In the ISO 7029 data, males aged from 18 to 30 years, 31 to 40 years, 41 to 50 years, 51 to 60 years, 61 to 70 years and 71 to 80 years had median $\mathrm{AAHL}_{1,2,3}$ of 0 to $5 \mathrm{kHz}, 5$ to $10 \mathrm{kHz}$, 10 to $25 \mathrm{kHz}, 25$ to $40 \mathrm{kHz}, 40$ to $60 \mathrm{kHz}$ and 60 to $85 \mathrm{kHz}$, respectively. In comparison males in the 'overall occupational group without noise exposure' section of the NSH aged from 18 to 30 years, 31 to 40 years, 41 to 50 years, 51 to 60 years, 61 to 70 years and 71 to 80 years had mean $\mathrm{AAHL}_{1,2,3}$ of $3.6 \mathrm{kHz}, 6.7 \mathrm{kHz}$, $10.3 \mathrm{kHz}, 16.3 \mathrm{kHz}, 21.4 \mathrm{kHz}$ and $30.8 \mathrm{kHz}$, respectively (Figure 1a). Similarly, females in the ISO 7029 data aged from 18 to 30 years, 31 to 40 years, 41 to 50 years, 51 to 60 years, 61 to 70 years and 71 to 80 years had median $\mathrm{AAHL}_{1,2,3}$ of $0 \mathrm{kHz}, 5$ to $10 \mathrm{kHz}, 10$ to $25 \mathrm{kHz}, 25$ to $30 \mathrm{kHz}, 30$ to $45 \mathrm{kHz}$ and 50 to $685 \mathrm{kHz}$, respectively. In comparison, females in the 'overall occupational group without noise exposure' section of the NSH aged from 18 to 30 years, 31 to 40 years, 41 to 50 years, 51 to 60 years, 61 to 70 years and 71 to 80 years had a mean $\mathrm{AAHL}_{1,2,3}$ of $6.2 \mathrm{kHz}, 6.4 \mathrm{kHz}$, $9.1 \mathrm{kHz}, 14.3 \mathrm{kHz}, 19.1 \mathrm{kHz}$ and $30.8 \mathrm{kHz}$, respectively (Figure 1b).

Overall, 101 ears $(73.7 \%)$ had a higher threshold than the ISO 7029 median $\mathrm{AAHL}_{1,2,3}$ which included 56 of 78 ears in the male group $(71.8 \%)$ and 45 of 59 ears in the female group (76.3\%) (Figure $1 \mathrm{a}$ and $1 \mathrm{~b}$, Table 1 ). There was no significant difference between the proportion of ears with thresholds higher than the ISO 7029 median $\mathrm{AAHL}_{1,2,3}$ on the same side as eyes without PXF, with PXF but no glaucoma and with PXF and glaucoma, in either the male or female groups (Table 1). In the 14 patients with unilateral PXF the hearing thresholds and their relation to the ISO 7029 median $\mathrm{AAHL}_{1,2,3}$ was similar in both ears.

\section{Discussion}

Previous studies, which used a variety of inclusion criteria and examination methods, have been inconclusive regarding the relation between hearing loss and glaucoma. ${ }^{12-15}$ One of these studies found no significant difference in hearing thresholds between patients with glaucoma and population-based
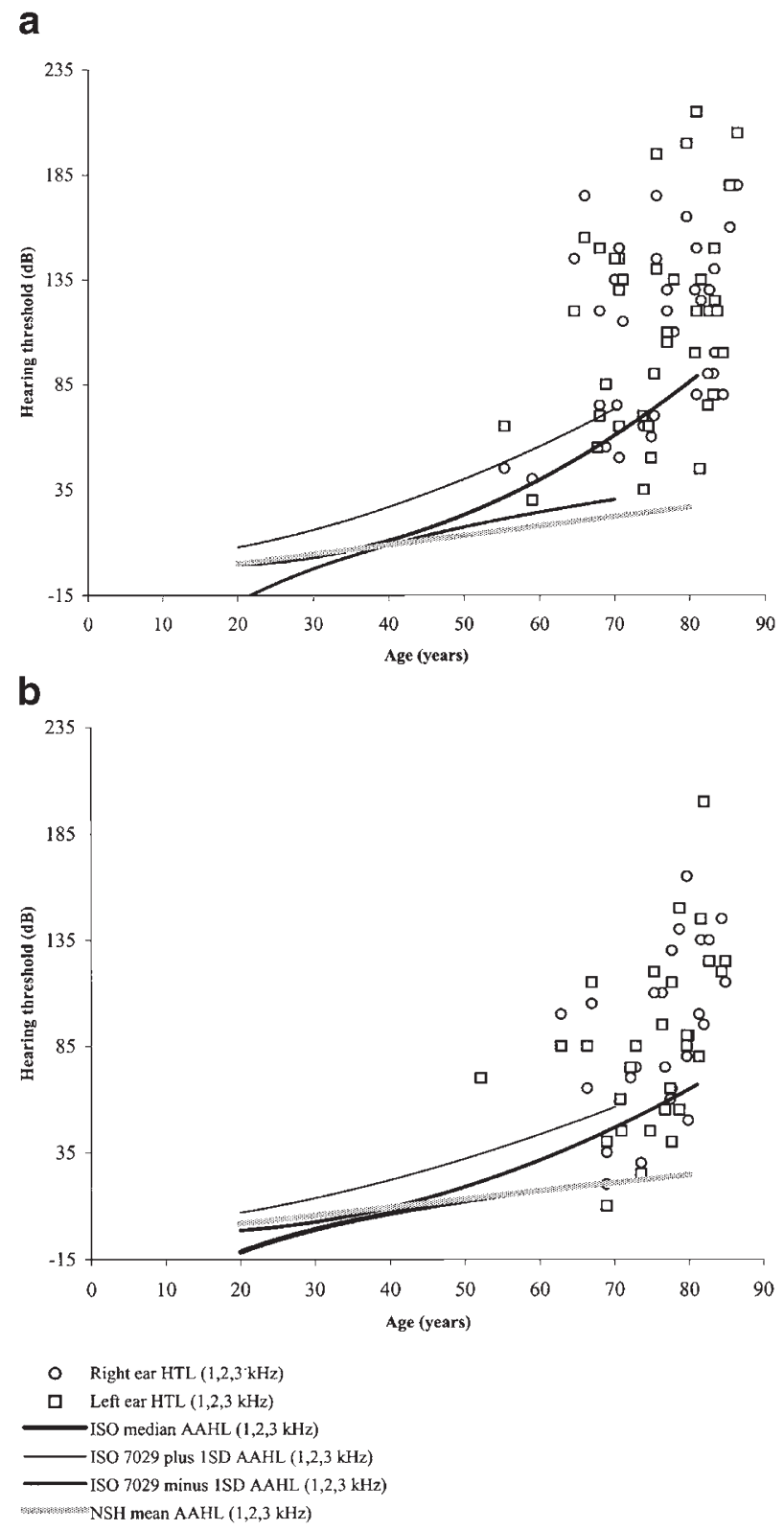

Figure 1 (a) Pseudoexfoliation and sensorineural hearing loss. Male right and left ear $\mathrm{HTL}_{1,2,3}$ compared with ISO 7029 median $\mathrm{AHHL}_{1,2,3}$ and NSH mean $\mathrm{AHHL}_{1,2,3}$. (b) Pseudoexfoliation and sensorineural hearing loss. Female right and left ear $\mathrm{HTL}_{1,2,3}$ compared with ISO 7029 median $\mathrm{AHHL}_{1,2,3}$ and $\mathrm{NSH}$ mean AHHL $_{1,2,3}$.

standards, but glaucoma secondary to pseudoexfoliation was not included as a distinct group. ${ }^{12}$ Up to $50 \%$ of eyes with PXF will develop glaucoma and the even higher prevalence of patients with PXF and glaucoma in this study probably reflects a selection bias, as our study was hospital based.,3,19,20 We would agree however, that glaucoma itself is not 
Table 1 Pseudoexfoliation syndrome and sensorineural hearing loss. Results of hearing tests in ipsilateral ears

\begin{tabular}{|c|c|c|c|c|}
\hline & $\begin{array}{c}\text { Eyes without } \\
\text { PXF }\end{array}$ & $\begin{array}{l}\text { Eyes with PXF } \\
\text { without glaucoma }\end{array}$ & $\begin{array}{c}\text { Eyes with PXF with } \\
\text { glaucoma }\end{array}$ & $P$ value \\
\hline \multicolumn{5}{|l|}{ Male patients } \\
\hline Total no. of ears tested & 7 & 17 & 54 & \\
\hline No $(\%)$ above ISO 7029 median $\mathrm{AAHL}_{1,2,3}{ }^{\mathrm{a}}$ & $6(85.7)$ & $11(64.7)$ & $39(72.2)$ & 0.58 \\
\hline \multicolumn{5}{|l|}{ Female patients } \\
\hline Total no. of ears tested & 7 & 12 & 40 & \\
\hline No $(\%)$ above ISO 7029 median $\mathrm{AAHL}_{1,2,3}$ & $5(71.4)$ & $8(66.6)$ & $32(80.0)$ & 0.60 \\
\hline
\end{tabular}

a $\mathrm{AAHL}_{1,2,3}$, age-associated hearing loss summed over 1, 2 and $3 \mathrm{kHz}$.

associated with hearing loss as the proportion of ears with mean hearing thresholds greater than the population standard was the same in both PXF groups, regardless of whether or not the eye ipsilateral to the tested ear had glaucoma. A study of consecutive patients such as our study has limitations and a larger, prospective, case control study of hearing thresholds in patients with primary open angle glaucoma may be warranted to clarify the relation between glaucoma and hearing loss.

Estimation of normal hearing loss with increasing age is notoriously difficult to determine. A number of population-based studies have provided data on the normal reduction in hearing associated with ageing in both otologically normal subjects and in typical populations. ${ }^{9-11}$ The studies based on otologically normal people have the advantage of being based on very large sample populations but also have a number of inherent problems. ${ }^{9,11}$ These include imprecise definitions of what constitutes otologically normal, variations in the audiometric techniques and bias in the recruitment process to select test subjects. The studies based on more typical populations have the disadvantage of smaller numbers of subjects, but were carefully designed to reduce selection bias and include all types of subjects to produce a more realistic assessment of normal hearing loss. ${ }^{10}$ Interestingly, a comparison of data from ISO 7029 of otologically normal subjects and the NSH based on a typical population, demonstrated about a $1-\mathrm{dB}$ difference in AAHL over a range of ages in males and a difference of only $3 \mathrm{~dB}$ in females. ${ }^{9}$ Furthermore, the hearing thresholds for older patients are in fact lower in the NSH than they are in the ISO 7029 data, which would result in a higher proportion of our patients having abnormal hearing when compared with age-matched controls if we used the NSH data. ${ }^{9,10}$ The optimal combination of frequencies used during audiometry to assess patients and controls is also controversial. ${ }^{9,10}$ Our decision to use 1, 2 and $3 \mathrm{kHz}$ as descriptors was based on the availability of control data and comparative data showing no difference between this combination and the combination of $0.5,1,2$ and $4 \mathrm{kHz}$ in assessing hearing disability. ${ }^{9,10}$

We believe that the presence of PXF, rather than glaucoma, is associated with hearing loss. It is possible that PXF is a systemic condition although there are conflicting reports regarding its association with serious disease. One recent epidemiological study found no association between ocular PXF and mortality, although serious disorders have multifactorial aetiologies and no data regarding confounding factors were used in the analysis. ${ }^{21} \mathrm{~A}$ second study demonstrated that patients with primary open angle glaucoma had a higher prevalence of diabetes than those with PXF, but diabetes is an extremely common disorder and the numbers in that study were small. ${ }^{22}$ Conversely, identical fibrils to those seen in PXF have been histologically demonstrated in the basement membranes and extracellular matrices of extraocular orbital tissues, the skin and visceral organs. ${ }^{4-6}$ Furthermore, PXF has also been associated with an increased risk of vascular disease, which may be due to the presence of PXF fibrils in the walls of blood vessels. ${ }^{23}$ If ocular PXF is indeed a sign of widespread distribution of PXF fibrils, deposition of fibrils in the inner ear may explain the association between PXF and hearing loss.

The development and function of the inner ear among vertebrates is remarkably similar and most of the understanding regarding both the development and function of inner ear structures is derived from animal models. ${ }^{24}$ Embryologically, the anterior segment structures of the eye affected by PXF and the tectorial and basilar membranes in the inner ear are both derived from neural ectoderm. ${ }^{25}$ The structure of both the tectorial and basilar membranes includes predominantly type II collagen, with each having different amounts of associated proteoglycan/ glycoprotein matrix. ${ }^{26}$ Interestingly type IV collagen 
fibres similar to those seen predominantly in the lens capsule have been isolated in both the tectorial and basilar membranes of an animal model. ${ }^{27}$ Besides its primary mechanical function, the tectorial membrane appears to be involved in controlling the chemical microenvironment of the stereocilia. ${ }^{78}$ Furthermore, the auditory hair cells appear to be actively involved in mechanically tuning the basilar membrane. ${ }^{7,8}$ It is possible that the apparent association between PXF and elevated hearing threshold seen in this study is due to the presence of PXF fibrils in the organ of Corti. Structural alteration of either (or both) the tectorial and basilar membranes by deposition of PXF fibrils could change the way in which vibratory energy is conducted to the sensory hair cells and alter their surrounding chemical environment resulting in sensorineural hearing loss. ${ }^{26,27}$

Interestingly, the majority of ears ipsilateral to eyes without PXF also had elevated hearing thresholds. Examination of the laterality of hearing loss with regard to the presence of PXF in the ipsilateral eye may be artificial in view of the small numbers in this study. It is possible that this finding was due to chance, but to our knowledge no previous study has demonstrated similar findings. However, two recent studies have suggested that PXF may be present in an eye without clinically visible deposits on the lens capsule and this can be demonstrated histologically. ${ }^{1,28}$ Furthermore, patients with PXF initially involving one eye can develop clinical signs in the second eye if followed up, and these cases of PXF may be more asymmetric than monocular. ${ }^{28,29}$ All patients in our study had at least one eye with PXF and given its probable widespread distribution in basement membranes it may be deposited in the inner ear before or independently of any manifestation in the anterior segment structures. A longitudinal study of these particular patients may help clarify this point.

\section{Conclusions}

A large proportion of patients with PXF have sensorineural hearing loss in comparison to agematched controls, regardless of whether or not this is associated with glaucoma. This finding supports the theory that PXF may be a systemic condition.

\section{References}

1 Ritch R. Exfoliation syndrome. Focal Points 1994; 12: 1-12.

2 Forsius H. Exfoliation syndrome in various ethnic populations. Acta Ophthalmol 1988; 66 (Suppl 184): 71-85.

3 Mitchell P, Wang JJ, Hourihan F. The relationship between glaucoma and pseudoexfoliation. The Blue
Mountains Eye Study. Arch Ophthalmol 1999; 117: 13191324.

4 Streeten BW, Dark AJ, Wallace RN, Li Z-Y, Hoepner JA Pseudoexfoliative fibrillopathy in the skin of patients with ocular pseudoexfoliation. Am J Ophthalmol 1990; 110: 490499.

5 Schlotzer-Schrehardt UM, Koca MR, Naumann GO, Volkholz H. Pseudoexfoliation syndrome. Ocular manifestation of a systemic disorder? Arch Ophthalmol 1992; 110: 1752-1756.

6 Streeten BW, Li Z-Y, Wallace RN, Eagle RC, Keshgegian AA. Pseudoexfoliative fibrillopathy in visceral organs of a patient with pseudoexfoliation syndrome. Arch Ophthalmol 1992; 110: 1757-1762.

7 Lim DJ. Functional structure of the organ of Corti: a review. Hear Res 1986; 22: 117-146.

8 Tsuprun V, Santi P. Ultrastructural organisation of proteoglycans and fibrillar matrix of the tectorial membrane. Hear Res 1997; 110: 107-118.

9 King PF, Coles RR, Lutman ME, Robinson DW. Assessment of Hearing Disability. Guidelines for Medicolegal Practice. Whurr: London, 1992.

10 Davis AC. Hearing in Adults. Whurr: London, 1995.

11 Shipton MS. Tables relating pure-tone audiometric threshold to age. In: Shipton MS (ed). Acoustics Report ac 94. National Physical Laboratory: Teddington, 1979.

12 Shapiro A, Siglock TJ, Ritch R, Malinoff R. Lack of association between hearing loss and glaucoma. Am J Otol 1997; 18: 172-174.

13 Seth RR, Dayal D. Inner-ear involvement in primary glaucoma. Ear Nose Throat J 1978; 57: 355-359.

14 Chilaris G, Coyas A. The occurrence of recruitment in glaucoma patients. J Laryngol Otol 1961; 75: 501-503.

15 Bietti GB. The result of audiometric examinations in some ocular diseases. Trans Ophthalmol Soc UK 1952; 72: 343355.

16 Gorlin RJ, Toriello HV, Cohen MM et al. Hereditary Hearing Loss and its Syndromes. Oxford University Press: New York, 1995.

17 Adoki II, Stoodley BJ. Abdominal aortic aneurysm, glaucoma and deafness: a new familial syndrome. $\mathrm{Br} \mathrm{J}$ Surg 1992; 79: 637-638.

18 Rundle P, Lottery AJ, Archer DB, McGinnity FG. Familial deafness associated with iris degeneration and glaucoma. Eye 1997; 11: 476-478.

19 Yanoff M. Intraocular pressure in exfoliation syndrome. Acta Ophthalmol 1988; 66 (Suppl): 59-61.

20 Montanes JM, Paredes AA, Garcia SC. Prevalence of pseudoexfoliation syndrome in the northwest of Spain. Acta Ophthalmol 1989; 67: 383-385.

21 Ringvold A, Blika S, Sandvik L. Pseudo-exfoliation and mortality. Acta Ophthalmol 1997; 75: 255-256.

22 Konstas AG, Tsatsos I, Kardasopoulos A, Bufidis T, Maskaleris G. Preoperative features of patients with exfoliation glaucoma and primary open-angle glaucoma The AHEPA study. Acta Ophthalmol 1998; 76: 208-212.

23 Mitchell P, Wang JJ, Smith W. Association of pseudoexfoliation syndrome with increased vascular risk. Am J Ophthalmol 1997; 124: 685-687.

24 Torres M, Giraldez F. The development of the vertebral inner ear. Mech Dev 1998; 71: 5-21.

25 Larsen WJ. Development of the head, the neck and the eyes and ears. In: Essentials of Embryology. Churchill Livingstone: New York, 1998, pp 229-272. 
26 Thalmann I. Collagen of accessory structures of the organ of corti. Connect Tiss Res 1993; 29: 191-201.

27 Kalluri R, Gattone VH 2nd, Hudson BG. Identification and localisation of type IV collagen chains in the inner ear cochlea. Connect Tiss Res 1998; 37: 143-150.

28 Kivela T, Hietanen J, Uusitalo M. Autopsy analysis of clinically unilateral exfoliation syndrome. Invest Ophthalmol Vis Sci 1997; 38: 2008-2015.

29 Layden WE. Exfoliation syndrome. In: Ritch R, Shields MD (eds). The Secondary Glaucomas. CV Mosby: St Louis, 1982, pp 99-120. 